Research Article

\title{
A questionnaire based survey to evaluate the usage pattern of carbapenems for sepsis management in intensive care unit
}

\author{
Vikram Kumar Shetty ${ }^{1}$, Vishwaprakash M. K. ${ }^{2}$, Somashekara S. C. ${ }^{2}$ \\ ${ }^{1}$ Department of Pharmacology, \\ Srinivas Institute of Medical \\ Sciences and Research Centre, \\ Mukka, Surathkal, Karnataka, \\ India, \\ ${ }^{2}$ Department of Pharmacology, \\ Malabar Medical College and \\ Research Institute, Modakkallur- \\ 673315, Kozhikode, Kerala, \\ India \\ Received: 25 December 2015 \\ Accepted: 03 February 2016 \\ *Correspondence to: \\ Dr. Vishwaprakash M. K., \\ Email: vishwapujar79@ \\ gmail.com

\begin{abstract}
Background: Sepsis remains one of the most common critical illnesses for patients to be admitted to an intensive care unit (ICU) and has very high associated morbidity and mortality ranging from 18 to $50 \%$. It is a well-known fact that early goal directed therapy with broad spectrum antibiotics, IV fluids and other supportive therapies improve outcome. The current study was done to know the importance of carbapenems in the management of sepsis focusing on perceived use and the clinical outcome in real time setting in tertiary care hospitals.
\end{abstract} \\ Methods: A structured questionnaire designed to collect information on the \\ perceived use of carbapenems, the clinical outcome, response period for clinical \\ cure and combination antibiotic therapy preferred with carbapenem was \\ circulated among 50 clinicians in tertiary care hospitals and nursing homes in \\ and around Mangalore between January 2015 to March 2015. \\ Results: Majority of doctors (>80\%) felt that carbapenems a broad spectrum \\ antibiotic administration provided good clinical improvement in hospital \\ infections along with improvement in blood cell count and bacteriological cure. \\ Copyright: (C) the author(s), \\ publisher and licensee Medip \\ Academy. This is an open- \\ access article distributed under \\ the terms of the Creative \\ Commons Attribution Non- \\ Conclusions: Our study provides an important insight regarding the knowledge, \\ practice pattern and clinical outcome through the use of carbapenem in Sepsis \\ management in ICU's around Mangalore. The experience of practice patterns \\ and clinical outcomes with carbapenem shared by doctors through this survey is \\ similar to that in other studies.
} Commercial License, which permits unrestricted noncommercial use, distribution, and reproduction in any medium, provided the original work is properly cited.

Keywords: Carbapenems, Sepsis, Early goal directed therapy, Antibiotic policy, Bacteriological cure

\section{INTRODUCTION}

Sepsis remains one of the most common critical illnesses for patients to be admitted to an intensive care unit (ICU). It is a well-known fact that late diagnosis often results in delayed treatment initiation leading to increased death of thousands of patients suffering from sepsis. Morbidity and mortality due to sepsis ranges from 18 to $50 \%{ }^{1}$ Patients suffering from pneumonia, bowel perforation, urinary infection or severe skin infections are at high risk of developing sepsis. In sepsis, gram-negative bacilli predominate, followed by gram-positive cocci and fungal species, especially Candida spp. The burden of severe sepsis in Indian ICUs could be majorly reduced by managing these infections. Figure 1 shows the distribution of major pathogens in sepsis in India. ${ }^{2}$
Successful management of sepsis with decreased morbidity and mortality rate requires integrated approach with the use of rigorous diagnostic measures, rapid initiation of appropriate antimicrobial/antibiotic therapy and supportive care. ${ }^{3}$ Antibiotic therapy remains the corner stone in the management of sepsis. The development of shock in life threatening conditions could be prevented by initiating empiric antibiotic treatment. ${ }^{4}$ Many studies have concluded that prompt initiation of antimicrobial therapy active against the causative pathogen is lifesaving in patients with severe sepsis., The surviving sepsis campaign strongly recommends initiating antibiotic therapy within the first hour of recognition of severe sepsis, after suitable samples have been obtained for culture. Early goal-directed therapy has 
significant positive impact on the outcome of severe sepsis and sepsis shock patients. ${ }^{6}$

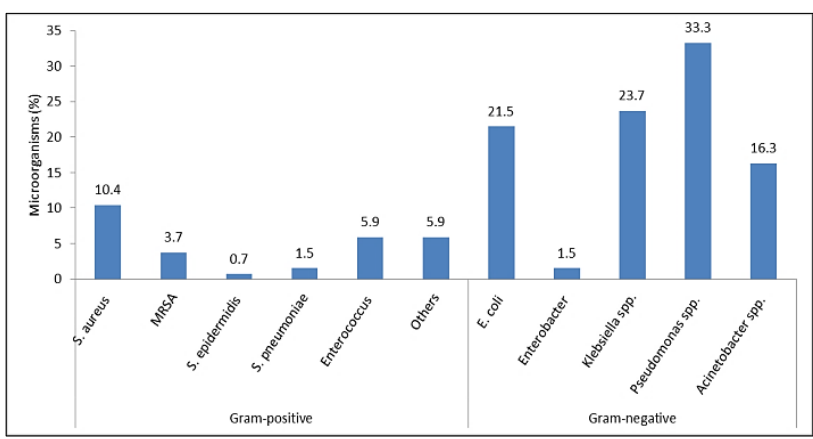

Figure 1: Major pathogens isolated from Indian sepsis patients.

Carbapenems are broad-spectrum agents that are useful in the empirical treatment of sepsis and other serious infections. Carbapenems are active against many clinically important pathogens and are particularly stable to a wide variety of pathogens producing $\beta$-lactamases (ESBLs and AmpC-type enzymes). As a result, they retain activity against a wide variety of resistant pathogens and this is of importance, as the incidence of resistant strains expressing ESBLs is increasing. ${ }^{7-10}$

Few data are available about the in-hospital experience of clinicians treating sepsis, on their perceived use of carbapenems in sepsis, prescription pattern and clinical outcome with the use of carbapenems in tertiary care hospital for sepsis management. This study was undertaken with the objective of knowing the importance of carbapenems in the management of sepsis focusing on perceived use and the clinical outcome in real time setting in intensive care in and around Mangalore, India.

\section{METHODS}

This study is a cross-sectional questionnaire based survey among clinicians who managed sepsis in intensive care unit in hospitals in and around Mangalore. Mangalore is one of the district places in Karnataka state, southern India. The study was conducted between January 2015 to March 2015. Ethical clearance was obtained by the institutional ethics committee. The questionnaire (Figure 2) was distributed among clinicians who managed sepsis in intensive care in hospitals to share their real time experience with carbapenems in management of sepsis. The questionnaire was designed to collect information on the experience of clinicians on their perceived use of carbapenems, the clinical response in patients after carbapenem use, response period for clinical improvement after carbapenem therapy, the duration of carbapenem therapy and other antibiotics given along with carbapenems in management of sepsis. The clinicians completed these questionnaires and handed back to us within 7 days. The responses were collated, analyzed and the results compared with that of published studies. The following were the parameters evaluated in this study:

- Preferred clinical conditions where carbapenems are used the most

- Clinical, bacteriological cure and blood cell count following carbapenem therapy

- Time to response after initiation of carbapenem therapy

- Duration to achieve best results following carbapenem therapy

- Effect of carbapenem therapy on hospital/ICU stay and response to add on therapy preferred with carbapenems

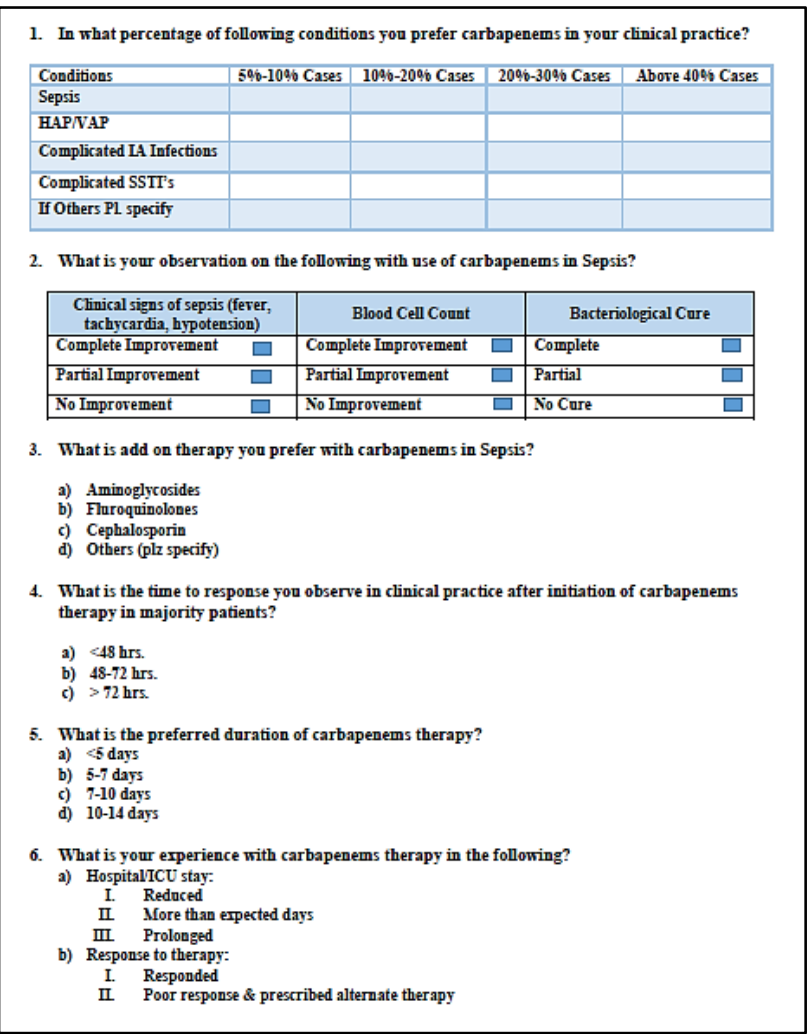

Figure 2: In -hospital clinic experience sharing on the usage pattern of carbapenem ICU.

\section{RESULTS}

A total of 50 clinicians managing Sepsis in tertiary hospitals around Mangalore responded to the questionnaire. The data collected and analysed, the clinical response in sepsis patients after carbapenem use, time for clinical improvement and preferred combination antibiotic therapy with carbapenems.

In our survey more than $74 \%$ of doctors used carbapenem for treating sepsis in more than $40 \%$ of cases. This was followed by hospital acquired pneumonia/ventilator associated pneumonia, cIAIs, cSSTIs and other infections that include CAP and lower respiratory tract infections (LRTIs) (Figure 3). 


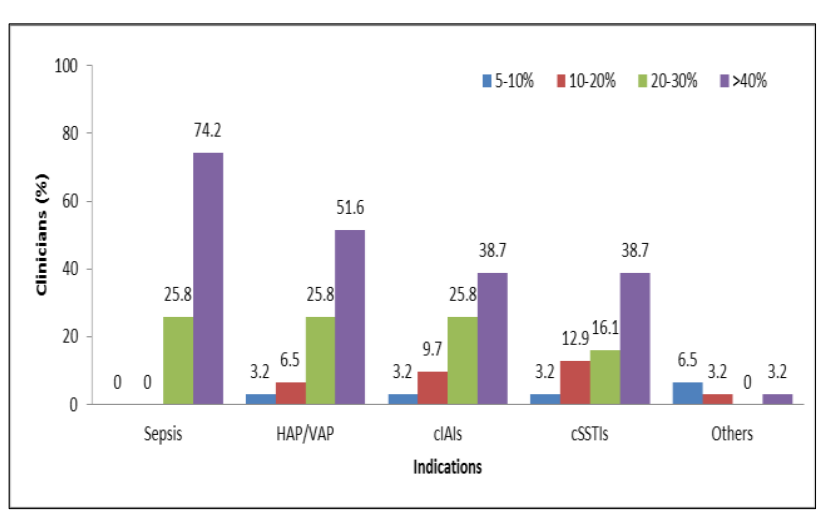

Figure 3: Major usage pattern of carbapenems in ICU with different clinical conditions.

Majority of clinicians (>80\%) agreed that carbapenem administration showed clinical improvement in majority of sepsis patients. Eighty percent of clinicians observed that carbapenem provided complete bacteriological cure and improvement in blood cell counts (Figure 4). About seventy percent of clinicians reported that majority of their patients responded to carbapenem therapy within 48-72 hours of initiation of therapy, while few have reported that patients responded within $<48$ hours and $>72$ hours following therapy (Figure 5).

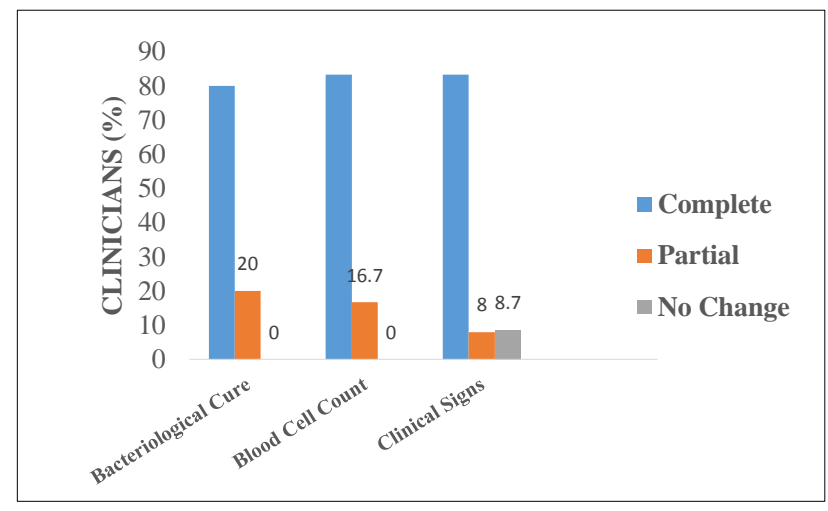

Figure 4: Clinician's perception on the patient's clinical improvement following carbapenem therapy.

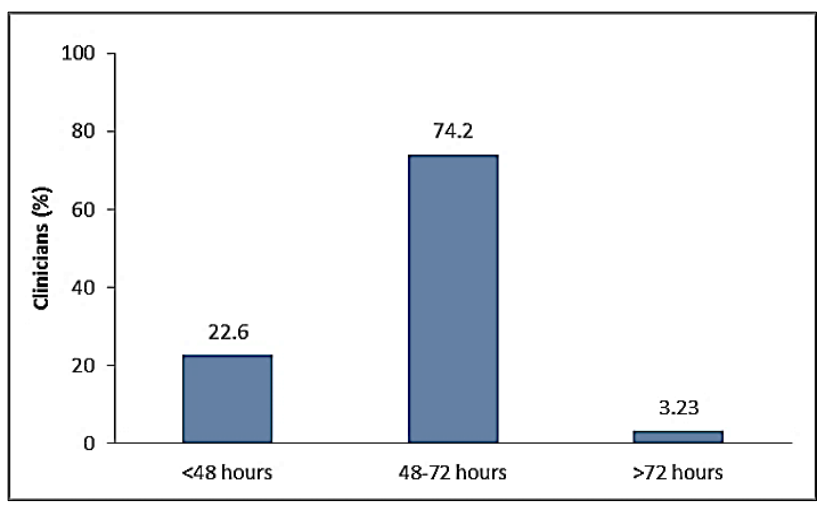

Figure 5: Clinician's perception on time taken for clinical improvement following carbapenem therapy.
Majority of clinicians (80\%) opined that carbapenem administration showed clinical improvement in majority of sepsis patients within 7 to 10 days and few of the patients responded after 10 days.

Clinicians' preference on combination therapy with carbapenems

Majority of clinicians $(60 \%)$ used aminoglycosides along with carbapenems in managing sepsis. Aminoglycosides were followed by cephalosporins, fluoroquinolones and other antimicrobials such as vancomycin, colistin, teicoplanin, linezolid and metronidazole, either alone or in combination.

\section{DISCUSSION}

Carbapenems which have both gram positive and gram negative coverage is one of the broad spectrum antibiotics available for current therapy against sepsis. What makes them more attractive is there, safety profile and them being more effective in treating severe infections at varied sites. Their efficacy can be attributed to them being Zwitterions, having high affinity for penicillin binding protein from a broad range of bacteria and they are resistant to most of the $\beta$ lactamase enzymes from both gram positive and gram negative bacteria's. ${ }^{11}$ They are promising class of antimicrobial agents for the management of multi-resistant gram-negative bacilli especially in ICUs where extensive use of cephalosporins has led to rapid emergence of gram-negative resistance. In a study conducted in neonatal ICU on patients with severe infections due to Acinetobacter baumannii and Klebsiella pneumoniae an overall clinical and bacterial response was obtained in $94.3 \%$ of cases treated with carbapenems. ${ }^{12}$ Similarly in this study the doctors observed good clinical outcome, reduction in blood cell count and bacteriological cure in majority of patients.

As per surviving sepsis guideline, early goal directed therapy with empirical initiation of broad spectrum antibiotics provide good clinical outcome and reduce risk of mortality. Clinical improvement generally becomes apparent after first 48-72 hours of therapy and therefore the selected antibiotic should not be changed during this period unless progressive deterioration occurs or initial microbiological studies dictate so. ${ }^{13}$ In our study majority of doctors surveyed opined that their patients showed clinical improvement between 48 to 72 hours after initiation of antibiotics, thereby reducing hospital stay and health care cost.

Clinical data suggests that the treatment of resistant bacterial strains could be accomplished by the use of carbapenems such as meropenem either alone or in combination with aminoglycosides and fluoroquinolones. Depending on the culture and susceptibility other combinations have also been effective in the management of sepsis such as metronidazole, colistin etc. ${ }^{14,15}$ The survey showed that due to the high risk of mixed 
infections predominantly gram negative pathogens in sepsis patients, majority of doctors preferred to use aminoglycosides which has gram negative pathogen coverage along with carbapenems. This is followed by cephalosporins, fluoroquinolones, colistin etc. in combination with carbapenems. The trend of combination therapy is similar to practice seen in other hospitals across.

The duration of antibiotic therapy for carbapenem depends on clinical response of the patient. Most of the doctors $(80 \%)$ preferred to use carbapenem for 7 to 10 days and only few patients 'needed therapy beyond 10 days. Complete duration of antibiotic therapy is important to prevent antibiotic resistance.

\section{CONCLUSIONS}

Sepsis and septic shock are important causes of morbidity and mortality in children, adults, elderly, surgical patients and trauma victims. Key to a successful outcome for patients with severe sepsis is prompt identification and early aggressive treatment, early goal-directed therapy can decrease mortality rate. Carbapenems having broad spectrum action is commonly used as an empirical therapy to have better clinical outcome in sepsis management.

Our study provides an important insight regarding the knowledge, practice pattern and clinical outcome through the use of carbapenem in sepsis management in ICU's around Mangalore. The experience of practice pattern and clinical outcome with carbapenems shared by doctors through this survey is similar to other hospitals as published in other studies. We recommend continuous knowledge updating program on antibiotic use, strong antibiotic policy and improved microbiology support in their hospitals will prevent antibiotic misuse in future.

\section{Funding: No funding sources}

Conflict of interest: None declared

Ethical approval: The study was approved by the Institutional Ethics Committee

\section{REFERENCES}

1. Levinson AT, Casserly BP, Levy MM. Reducing mortality in severe sepsis and septic shock. Available at http://www.medscape.com/ viewarticle/ 749208. Accessed on 23 December 2015 at 12pm.

2. Divatia JV. Reducing the burden of severe sepsis and infections in Indian ICUs. Available at http: //goo.gl/12Dbhv. Accessed on December 18, 2014.
3. Bochud PY, Glauser MP, Calandra T. Antibiotics in sepsis. Intensive Care Med. 2001;27:S33-48.

4. Ferrer R, Artigas A, Suarez D, Palencia E, Levy MM, Arenzana A, et al. Effectiveness of treatments for severe sepsis: a prospective, multicenter, observational study. Am J Respir Crit Care Med. 2009;180:861-6.

5. Garnacho-Montero J, Garcia-Garmendia JL, Barrero-Almodovar A, Jimenez Jimenez FJ, PerezParedes C, Ortiz-Leyba C. Impact of adequate empirical antibiotic therapy on the outcome of patients admitted to the intensive care unit with sepsis. Crit Care Med. 2003;31:2742-51.

6. Rivers E, Nguyen B, Havstad S, Ressler J, Muzzin A, Knoblich B, Peterson E, Tomlanovich M. Early goal-directed therapy collaborative group. Early goal directed therapy in the treatment of severe sepsis and septic shock. N Engl J Med. 2001;345:1368-77.

7. Bush K. New $\beta$-lactamases in gram-negative bacteria: diversity and impact on the selection of antimicrobial therapy. Clin Infect Dis. 2001;32:1085-9.

8. Witte W, Mielke M. $\beta$-Laktamasenmitbreitem Wirkungsspektrum. BundesgesundheitsblGesundheitsforsch-Gesundheitschutz. 2003;46:88190.

9. Jacoby GA, Munoz-Price LS. The new betalactamases. N Engl J Med. 2005;352:380-91.

10. Paterson DL, Bonomo RA. Extended-spectrum blactamases: a clinical update. Clin Microbiol Rev. 2005;18:657-86.

11. Kattan JN, Villegas MV, Quinn JP. New developments in carbapenems. Clin Microbiol Infect. 2008;14:1102-11.

12. Köksal N, Hacimustafaoğlu M, Bağci S, cCelebi S. Meropenem in neonatal severe infections due to multiresistant gram-negative bacteria. Indian $\mathbf{J}$ Pediatr. 2001;68(1):15-9.

13. Guidelines for the management of adults with hospital-acquired, ventilator-associated, and healthcare-associated pneumonia. American J Respir Critl Care Med. 2005;171(4):388-416.

14. Duszyńska W. Strategies of empiric antibiotic therapy in severe sepsis. Anaesthesiol Intensive Ther. 2012;44(2):96-103.

15. Sundaram S. Antibiotic use in critical care. Available at www.nes.scot.nhs.uk/ media/ 2575124/ antibiotic_use_in_critical_care.ppt. Accessed on 20 December 2014 at $3 \mathrm{pm}$.

Cite this article as: Shetty VK, Vishwaprakash MK, Somashekara SC. A questionnaire based survey to evaluate the usage pattern of carbapenems for sepsis management in intensive care unit. Int J Basic Clin Pharmacol 2016;5:235-8. 